\title{
BMJ Open Assessing preferences for leisure activities of people receiving adult day services: a study protocol for concept mapping and psychometric testing
}

\author{
Mike Rommerskirch-Manietta (D) ,,2 Daniel Purwins, ${ }^{1,2}$ Kimberly Van Haitsma, ${ }^{3}$ \\ Katherine Abbott, ${ }^{4,5}$ Daniela Rodrigues-Recchia, ${ }^{1,2}$ Martina Roes (D) 1,2
}

To cite: RommerskirchManietta M, Purwins D, Van Haitsma K, et al. Assessing preferences for leisure activities of people receiving adult day services: a study protocol for concept mapping and psychometric testing. BMJ Open 2021;11:e055069. doi:10.1136/ bmjopen-2021-055069

- Prepublication history and additional supplemental material for this paper are available online. To view these files, please visit the journal online (http://dx.doi.org/10.1136/ bmjopen-2021-055069).

Received 03 July 2021 Accepted 22 October 2021

D) Check for updates

(c) Author(s) (or their employer(s)) 2021. Re-use permitted under CC BY-NC. No commercial re-use. See rights and permissions. Published by BMJ.

For numbered affiliations see end of article.

\section{Correspondence to} Mike Rommerskirch-Manietta; Mike.Rommerskirch-Manietta@ dzne.de

\section{ABSTRACT}

Introduction Leisure activities appear to be an important factor in maintaining and improving health in old age. To better understand what people want to do when visiting an adult day service (ADS), it is important to systematically assess their preferences. Currently, there is no instrument for assessing preferences for leisure activities for people receiving ADS. Accordingly, the planned study aims to develop or modify and psychometrically test an instrument to assess leisure activities preferences for use with people receiving ADS.

Methods and analysis A mixed-method design with a participatory research approach was chosen for this study (Preferences for Everyday Living-Deutschland, PELI-D II). In the first step of this study, leisure activities will be identified on the basis of an evidence map. In the second step, the results from the evidence map will be empirically supplemented, and leisure activities will be categorised and prioritised within a concept mapping approach by people who receive ADS. Subsequently, based on this categorisation, either an instrument that was piloted in a previous study (PELI-D I) will be modified or an instrument with a focus on preferences for leisure activities in ADS will be explored. In the last step of this study, the instrument will be psychometrically tested. Data will be analysed via content analysis as well as descriptive and inferential statistics and statistical tests. The results will be presented in various tables and graphs (eg, pattern matching).

Ethics and dissemination This study was approved by the ethics committee of the Witten/Herdecke University (application number 226/2020). The results will be made available to the public at (inter)national conferences, in peer-reviewed articles and in articles for practitioners.

\section{INTRODUCTION}

The organising and performing of leisure activities are associated with the maintenance or improvement of health in older people. ${ }^{1-3}$ Conceptually, these actions describe that the choice of which activities are understood and completed as leisure activities depends on, among other things, individual preferences. $^{3-5}$ For example, shopping can be
Strengths and limitations of this study

- The strength of this study will be that leisure activities from the perspective of people receiving adult day services (ADS) will be identified for the first time.

- Concept mapping represents also a participative research approach.

- In addition, the voice of the people receiving ADS will be valued because they are involved in data generation, data analyses and validation of the results.

- Challenges in recruiting people in ADS to participate may be encountered because of the time consuming concept mapping process.

- Ethical challenges in terms of the participation of people with dementia in the research process will be addressed through an ongoing/process consent approach.

viewed either as a necessary everyday activity or as a leisure activity. ${ }^{6}$ For people with care needs, participating in leisure activities is often associated with barriers, such as restrictions in everyday life associated with the need for care support. ${ }^{7}$ In congregate settings, such as adult day services (ADS), the preferences of the individuals are are often not considered. ${ }^{89}$ Thus, services that do not reflect the preferences of people receiving care often lead to a limited option of leisure activities. ${ }^{70-12}$ Organising leisure activities on the basis of individual preferences is relevant to ADS. However, the same leisure activities are often offered in the various ADS, for example, conversation and reading sessions, social games, music and dancing, physical and cultural activities. ${ }^{13}$ Although, the selection of these activities is less tailored to the respective people and not preference oriented. ADS is aimed at strengthening the daily structure of people receiving ADS who live at home and may additionally use home care services and/or social care-oriented 
services. ${ }^{13-15}$ In addition, ADS aimed also at supporting family caregivers to remain employed. ${ }^{16}$ Especially in times of the COVID-19 pandemic, the importance of ADS for older people with care needs and family caregivers has become clear. A survey of thirty ADS facilities in the USA ( $\mathrm{n}=22$ responded) found that $95.5 \% \quad(\mathrm{n}=21)$ of the participating facilities were closed in August 2020. As a result of this, an increase of social isolation of older people with care needs was reported, which was likely to be associated with a decline in their health, particularly in terms of cognition. In terms of family caregivers, stress and anxiety were reported to have increased significantly. One ADS in Ohio reported that $74 \%$ of family caregivers had to choose between continuing their job or caring for their relative. ${ }^{16}$ Despite these recent experiences, inconsistent results can be found for the effectiveness of ADS, for example, related to delaying the need for individuals to move into a nursing home. ${ }^{13} 1416$ For the systematic assessment of preferences of people, various instruments in different languages exist, that focus on different topics or care settings. ${ }^{17-20}$ However, according to an initial literature review conducted by the authors of this protocol, no instruments currently exist with an explicit broad focus on leisure activities in the ADS; therefore, what kind of preferences with regard to leisure activities people receiving ADS may have remains unknown. Furthermore, it is unknown how these individuals would categorise and prioritise leisure activities and whether an assessment of leisure preferences would even be feasible.

This study protocol refers to the follow-up study Preferences for Everyday Living-Deutschland (PELI-D) II (2020-2023), that builds on the findings of the PELI-D I study (2017-2020). In PELI-D I, an instrument for assessing preferences for everyday living was translated from American English into German and then piloted in various nursing care settings in Germany. ${ }^{18}$ 21-24 Researchers and stakeholders participating in PELI-D I identified the demand for an instrument to assess preferences for leisure activities for people receiving ADS. This demand was expressed due to practicability, applicationrelated reasons, care focus reasons and the current research gap (eg, scope of the questions, number of questions and specifics of the care setting).

As a result of this central finding, the PELI-D II study aims to identify and structure the leisure activities of people receiving ADS. The results will either lead to a modification of an existing instrument (PELI-D) or to the development of a new psychometrically tested instrument. ${ }^{18}$ In this study, leisure activities are preliminarily defined as activities in the context of ADS and will be defined in more detail within this study on the basis of an evidence map and the perspectives of people receiving ADS. The following three research questions will be addressed:

1. 'Which leisure activities of people receiving care are measured by existing instruments for assessing preferences for everyday living?'

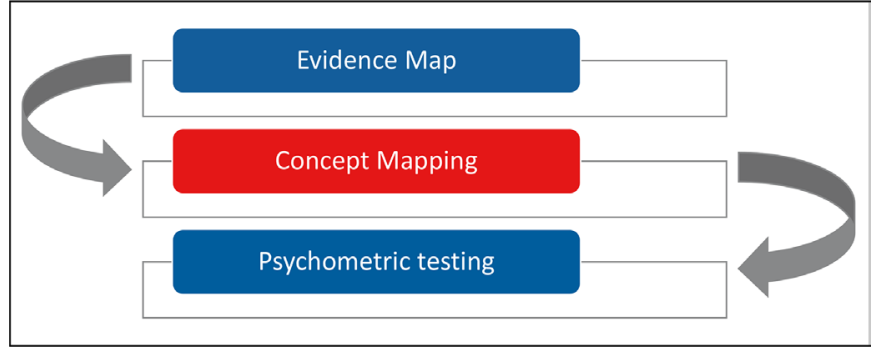

Participatory research approach

Considering results

Figure 1 Steps of the mixed-method research design with a participatory research approach.

2. 'Which preferences are described by people receiving ADS in relation to leisure activities, and how are leisure activities categorised and prioritised by them?'

3. 'What is the reliability of an instrument assessing preferences related to leisure activities for people receiving ADS?'

\section{METHODS AND ANALYSIS}

A mixed-method design is chosen, taking into account a participatory research approach (figure 1) to answer the three research questions. In the first step, an evidence map ${ }^{25}$ will be carried out to answer the first research question and as a basis for the concept mapping. ${ }^{26}$ In the second step, the results of the evidence map will be empirical supplemented by an concept mapping ${ }^{26}$ approach to answer the second research question. Finally, a psychometric test phase ${ }^{27}$ will be conducted to test the test-retest reliability of an instrument assessing preferences related to leisure activities of people receiving ADS. All data collections (concept mapping and psychometric testing) will be conducted in various ADS in the federal states of North Rhine-Westphalia and Rhineland-Palatinate in Germany.

For the reporting of this protocol, whenever applicable, the Standards for Reporting Qualitative Research will be followed (online supplemental table 1). ${ }^{28}$

\section{Participatory research approach}

The degree of participation of people in research is divided into four basic levels (figure 2) ${ }^{29}$ It is important to note that participation is not an either/or option. Rather, participation is a developmental process, the implementation of which depends on the conditions of the study and the living conditions of the target group. ${ }^{30}$

For the PELI-D II study, the cognitive conditions of potential participants need to be taken into account. Therefore, aspects of the preliminary stage of participation (hearing the voices of and involving the people who receive ADS) and the stage of participation (codetermination and partial decision-making authority) will be considered (figure 2). This implementation will be performed within the framework of the individual steps of concept mapping. 


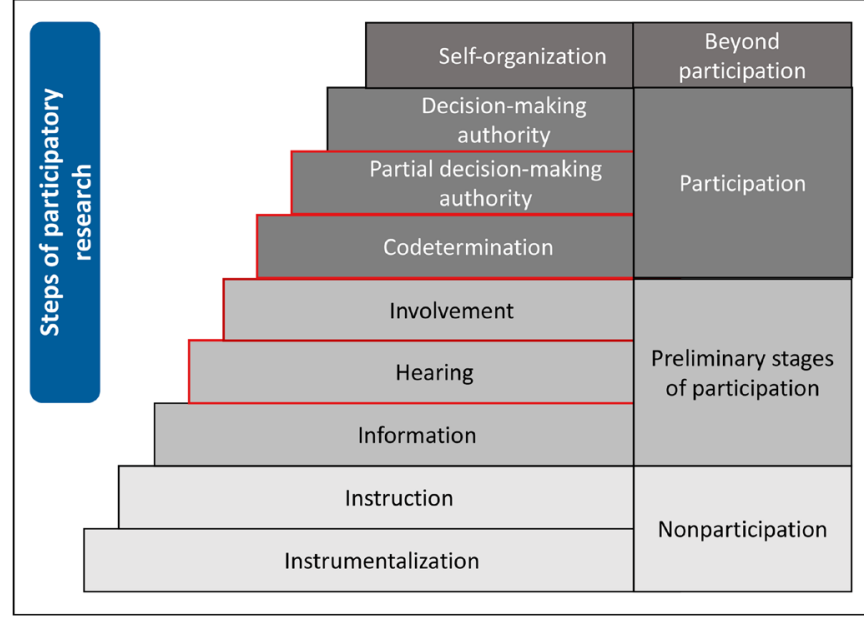

Planned consideration of the steps of

participatory research in the project PELI-D II

Figure 2 Steps of participatory research. PELI-D,

Preferences for Everyday Living-Deutschland.

\section{Method addressing research question 1 \\ Evidence map}

For the identification of the leisure activities of people receiving care, which are assessed with instruments for assessing preferences for everyday living, a literature review will be conducted, starting in November 2020 and scheduled to end in August 2021, using an evidence map approach. ${ }^{25}$ According to the framework of Arksey and $\mathrm{O}^{\prime}$ Malley ${ }^{31}$ the following steps will be considered: (1) the identification of a broad (clinical) question, (2) the identification of relevant studies, (3) study selection, (4) presentation of the data, (5) the reporting of the data and (6) the sharing of and consultation regarding findings. ${ }^{31} 32$ More details about the evidence map (eg, databases, inclusion and exclusion criteria, search string) are reported in the review protocol. ${ }^{33}$

\section{Method addressing research question 2 \\ Concept mapping}

Concept mapping (figure 3) is scheduled to start in November 2021 and will end in May 2023. It follows the methodology and associated steps of Kane and Trochim. ${ }^{26}$ The six associated steps will be taken into account in the process of concept mapping (see pages 8-12). Furthermore, people receiving ADS will be involved in four of the six steps in a participatory way in this study.

\section{Method addressing research question 3}

\section{Psychometric testing}

For the psychometric testing of the modified or developed instrument to assess people's preferences, the stability of the items will be tested using test-retest reliability. ${ }^{27}$ This approach is essential for a systematic assessment of preferences by researchers and/or healthcare practitioners. ${ }^{27} 34$ Psychometric testing is scheduled for June 2023 and is expected to be completed in December 2023.

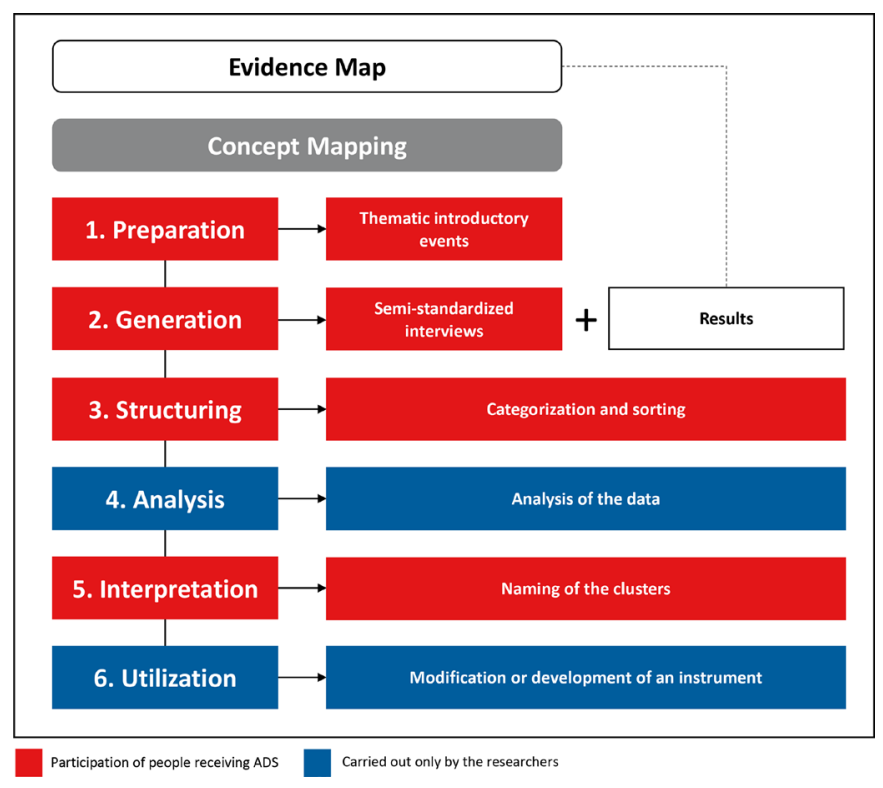

Figure 3 Process of concept mapping. ADS, adult day services.

\section{Sampling}

Field access to different ADS will be carried out with the support of the gatekeepers of different providers in the federal states of North Rhine-Westphalia and RhinelandPalatinate in Germany. Priority will be given to ADS that have already participated in the PELI-D I study conducted by the Deutsches Zentrum für Neurodegenerative Erkrankungen (DZNE) ${ }^{18}{ }^{35}$ This means that these providers already have initial experience participating in research studies and assessing preferences.

The concept mapping sample will include at least 10 individuals receiving ADS who are recruited from participating providers. ${ }^{26}$ People who receive ADS in Germany are characterised by the fact that they still live in their own homes and have care needs (limitations such as a need to structure their day). Nearly $60 \%$ of these people have been diagnosed with a geronto-psychiatric diseases or affective disorders such as dementia, depressive symptoms or other behavioural symptoms. The majority of people receiving ADS in Germany are female and on average 80 years old. ${ }^{13} 14$ These data can also be verified by the PELI-D I study, in which participants from the federal states of North Rhine-Westphalia and RhinelandPalatinate who received ADS showed the same characteristics. Regarding psychometric testing, once the instrument has been modified or developed, a calculation of the sample size for the test-retest reliability will be performed based on the minimum acceptable reliability (intraclass correlation coefficient value (ICC) $>0.6), 3637$ expected reliability (ICC) $(\rho 1)>0.75^{38}$,fixed power at 80 $\%$, alpha at 0.05 , expected dropout rate of $10 \%$ and the two measuring points $\left(\mathrm{t}_{0}-\mathrm{t}_{1}\right){ }^{39}{ }^{30}$

Inclusion criteria for both concept mapping and psychometric testing include: (1) all people receiving ADS at least once per week or four times per month, (2) individuals can verbally report on their preferences 
related to leisure activities and (3) sort their preferences displayed in the form of cards, pointing towards a certain preference and/or verbal cues.

The recruitment of people receiving ADS will be carried out in two ways: (1) The first way is primarily with the support of health professionals in the various participating ADS. Using their clinical competency assessment skills, these professionals will address ADS recipients who meet the inclusion criteria listed above and (2) In addition, people receiving ADS, their relatives and legal guardians will have the opportunity to contact the researchers (MR-M, DP and MR) directly and register their relative for the study and/or ask questions. Information materials (flyers and posters in elaborated plain language) ${ }^{41}$ will be distributed, an approach that is intended to draw the attention of people receiving ADS, their relatives and legal guardians and incentivise them to participate in the study.

\section{Data collection}

Data collection includes steps one through three of the concept mapping approach (preparation, generation and structuring; figure 3). Due to the COVID-19 pandemic, we will consider the hygiene protection guidelines established by participating facilities during our data collection period. In addition, the data collection process for psychometric testing is presented below.

\section{Step 1: preparation}

In the first step of concept mapping, kick-off meetings will be held in the respective ADS for participants. Here, an initial introduction to the topic of the study will take place. Additionally, the wishes and expectations of participants for concept mapping will be clarified and taken into account in the further planning of the study.

\section{Step 2: generation}

In the second step of the concept mapping, the different leisure activities will be generated for the structuring third step. For this purpose, the leisure activities identified based on the evidence map will be supplemented empirically and the understanding for the term leisure activities from the perspective of people receiving ADS will be investigated. Therefore, semistructured interviews will be conducted 'face to face' with the participants in the various ADS. ${ }^{42}$ The semistructured interviews will be conducted by a nursing scientist (MR-M) with a professional background as a geriatric nurse. In addition to his professional background the first author has also experience in interviewing older people with care needs with and without dementia. ${ }^{18}$ For these semistructured interviews, an interview guideline will be developed that takes the current literature on the topic of leisure activities into account. ${ }^{43}$ For example, we will ask the participants about former and current preferred leisure entertainment or relaxation activities. Additionally, we will ask them if they define their time spend in the ADS as leisure time and/or if activities provided at the ADS are understood as leisure activities. The interview guideline will be tested through a pretest and modified if necessary. Interviews will be audiorecorded and transcribed. ${ }^{44}$

\section{Step 3: structuring}

In preparation of the third step, all leisure activities identified with the evidence map and interviews will be written on cards. These cards will be created by three people of the research team (MR-M, DP and MR) and will be adapted to the needs of people receiving ADS, for example, feature a large font size, pictograms and elaborated plain language ${ }^{41}$ In the third step, the categorisation will be carried out by participants using these cards. ${ }^{26}$ The categorisation will be done based on the topic-related similarities of the different leisure activities to create content clusters. In addition, each leisure activity will be rated on a 5-point Likert scale according to the importance (relatively unimportant, somewhat important, moderately important, very important, extremely important) of this activity for the respective participant. Further, the top five leisure activities, which are most important to them personally, will be prioritised by the partcipants. ${ }^{45}$ The results from these sorting processes will be recorded by the researcher (MR-M) by taking pictures and then transferred to a software programme (Concept Systems). ${ }^{46}$

\section{Psychometric testing}

For the psychometric testing of the instrument modified or developed on the basis of concept mapping, a testretest approach will be carried out. ${ }^{27}$ For this purpose, data collection (for sample size calculation see sampling) with the instrument will take place at an interval of 1 week $\left(\mathrm{T}_{0}\right.$ and $\left.\mathrm{T}_{1}\right)$. All data collection for the testing of the instrument will be completed by the researcher (MR$\mathrm{M})$. The data collection will be carried out in an onsite computer-assisted manner in the respective ADS.

\section{Data analyses}

Steps four through six of the concept mapping (figure 3) will involve data analysis aspects and are presented below with the analyses of the psychometric testing data.

\section{Step 4: qualitative analysis}

The fourth step of concept mapping will be data analysis. For this purpose, the interviews will be first transcribed, pseudonymised and then imported into MAXQDA $2020{ }^{47}$ MAXQDA 2020 will be used within the study to analyse the transcribed interviews and in particular to categorise them. ${ }^{47}$ All authors have experience with the analysis of qualitative data. The subsequent data analysis will be carried out by two researchers (MR-M and MR) with the help of an inductive qualitative content analysis described by Elo and Kyngäs and Elo et al. ${ }^{48}{ }^{49}$ For this process, the steps open coding, creating categories and abstraction will be considered. In the first step (open coding), the transcribed interviews will be read several times by two researchers (MR-M and MR) and conspicuous points and headings will be noted using the memo function in MAXQDA 2020. In a following meeting, the 
two researchers will discuss their initial findings. Subsequently, the headings created with the memo function will be analysed separately by two researchers (MR-M and MR) and the first categories will be freely generated on the material. In the second step (category formation), the categories generated will be grouped independently by the same two researchers (MR-M and MR) under higherorder headings. The aim here is to reduce the number of categories, summarise similarities and to distinguish the categories from each other. In the last step (abstraction), the research topic will be described with the help of the categories formed. For this purpose, subcategories with similar content will be combined into categories and categories into main categories as long as seems reasonable and possible. The results of the two researchers (MR-M and MR) will be discussed and consensus defined. If necessary, the coding system will be recoded and the code system will be finalised. Finally, the results will be reflected on and discussed by the whole research team (MR-M, DP, MR, KA and KVH).

\section{Step 4: quantitative analysis}

The categorisations of the concept mapping process will be further analysed using Concept Systems, ${ }^{46}$ which allows for the calculation of the correlations between various sorted items. Subsequently, graphical processing will be conducted. For this purpose, a point map and a resulting cluster map will be created. ${ }^{45}$ In addition, a point rating and cluster rating map will be created based on the participants' rating of the different leisure activities. The analysis of the prioritisations will be done using both descriptive and inferential statistics. Different variants of tables and graphs (pattern match) will be used to present the results. ${ }^{45}$

\section{Psychometric testing}

Test-retest reliability will be analysed using R V.4.0.3. ${ }^{50}$ Statistical tests (eg, intraclass and Pearson's correlation coefficient, Cohen's kappa and Fisher's Z-transformation) will be taking into account the various sociodemographic characteristics of participants. ${ }^{51} 52$

\section{Step 5: interpretation}

In the fifth step, recruited participants will define the name of the identified clusters of sorted leisure activities, which will be done in the form of a participatory approach, together with the researchers (MR-M, DP and MR). For this purpose, the participants will be presented with a list of the leisure activities and their categorisation, which was created in the generation and structuring step (see pages 8-10). The participants will be asked to read through each of the individual leisure activities in the respective clusters and to name the cluster with a short phrase or word. Afterwards, all clusters will be discussed step by step individually by the participants presenting the cluster names. A consensus process will be conducted to agree on the cluster names. If there is no agreement, it will be possible to divide the clusters into subclusters. The researchers then present the point map to the participants. Here, the researchers point out that the statements are closer to each other, which were sorted together more often by the participants. The participants will be then taken on a 'tour' of the point map by the researchers and the individual leisure activities and their position on the map will be examined. Following this, the participants will be informed that the points have been grouped into clusters. These clusters were named by the participants in advance and will be now validated. The cluster map will be presented, and the participants will be informed by the researchers that clusters that are closer to each other should have greater similarity in terms of content than clusters that are further away. For this purpose, the participants will be again taken on a 'tour' of the cluster map and the individual clusters will be discussed and evaluated by the participants in terms of their content-related similarity regarding their respective distance from each other. In addition, the participants will be asked whether they can identify and name similar groups of clusters or regions. The next step will be the presentation of the point rating and the cluster rating map, which will be discussed and validated by the participants as part of a 'tour' of the maps. As a final step, the pattern match graphs will be presented by the researchers to provide the participants an insight into the degree of agreement between the subgroups and the ratings. Finally, these results will be discussed by the participants and the results will be recorded by the researchers. Step 5 takes place on site at the respective ADS. Due to the participants' care needs and related limitations, step 5 will be split up so that each discussion of the maps takes place on separate days.

\section{Step 6: utilisation}

For the sixth and final step of concept mapping, the modification or development of an instrument will be done based on the results of the entire concept mapping process. For this purpose, the structure and question wording of the translated instrument from the PELI-D I study will be used. ${ }^{18} 35$

\section{Patient and public involvement}

Due to the research design of this study, participants will be involved in the research process in a participatory way (figures 2 and 3). Especially in the concept mapping process, participants will be actively involved in different research steps and therefore can influence the process and results of this research.

\section{Ethics and dissemination}

For this study, the principles of the Declaration of Helsinki will be considered and applied. ${ }^{53}$ Participants will be informed about the study by means of informed consent in elaborated plain language ${ }^{41}$ and will only be able to participate in the study after they have given and signed consent. In addition, ongoing consent/process consent will be given during the course of the study. ${ }^{545}$ This means that participants will be asked at each step of 
concept mapping and/or psychometric testing whether they would like to continue participating in the study. ${ }^{55}$ Here, it is important that consent to participate in the study can be withdrawn by participants at any time. Furthermore, an ethical prognosis and ethical prevention will be made and carried out by the researchers (MR-M, DP and MR) in advance of the data collection. ${ }^{54}$ Regarding data protection, the collected data will be analysed pseudonymously and will be saved on passwordsecure servers.

An ethical proposal for this study has been submitted to the Witten/Herdecke University and has received ethical clearing (application number 226/2020). The results will be presented at (inter)national conferences and published in journals for practitioners as well as peerreviewed journals.

\section{Author affiliations}

${ }^{1}$ Deutsches Zentrum für Neurodegenerative Erkrankungen (DZNE), Witten, Germany ${ }^{2}$ Department of Nursing Science, Faculty of Health, Witten/Herdecke University, Witten, Germany

${ }^{3}$ College of Nursing, Penn State, University Park, Pennsylvania, USA

${ }^{4}$ Department of Sociology and Gerontology, Miami University, Oxford, Ohio, USA

${ }^{5}$ Scripps Gerontology Center, Miami University, Oxford, Ohio, USA

\section{Twitter Mike Rommerskirch-Manietta @_rochmro}

Contributors MR-M wrote the initial draft of the protocol. DP, KVH, KA, DR-R and MR revised the manuscript. KA, KVH and MR are supervisors of this study. All authors read and approved the final manuscript.

Funding The authors have not declared a specific grant for this research from any funding agency in the public, commercial or not-for-profit sectors.

Competing interests None declared.

Patient consent for publication Not applicable.

Provenance and peer review Not commissioned; externally peer reviewed.

Supplemental material This content has been supplied by the author(s). It has not been vetted by BMJ Publishing Group Limited (BMJ) and may not have been peer-reviewed. Any opinions or recommendations discussed are solely those of the author(s) and are not endorsed by BMJ. BMJ disclaims all liability and responsibility arising from any reliance placed on the content. Where the content includes any translated material, BMJ does not warrant the accuracy and reliability of the translations (including but not limited to local regulations, clinical guidelines, terminology, drug names and drug dosages), and is not responsible for any error and/or omissions arising from translation and adaptation or otherwise.

Open access This is an open access article distributed in accordance with the Creative Commons Attribution Non Commercial (CC BY-NC 4.0) license, which permits others to distribute, remix, adapt, build upon this work non-commercially, and license their derivative works on different terms, provided the original work is properly cited, appropriate credit is given, any changes made indicated, and the use is non-commercial. See: http://creativecommons.org/licenses/by-nc/4.0/.

\section{ORCID iDs}

Mike Rommerskirch-Manietta http://orcid.org/0000-0002-1533-3006

Martina Roes http://orcid.org/0000-0003-4531-8584

\section{REFERENCES}

1 Chang P-J, Wray L, Lin Y. Social relationships, leisure activity, and health in older adults. Health Psychol 2014;33:516-23.

2 Sala G, Jopp D, Gobet F, et al. The impact of leisure activities on older adults' cognitive function, physical function, and mental health. PLoS One 2019;14:e0225006.

3 Szanton SL, Walker RK, Roberts L, et al. Older adults' favorite activities are resoundingly active: findings from the NHATS study. Geriatr Nurs 2015;36:131-5.
4 Adams KB, Leibbrandt S, Moon H. A critical review of the literature on social and leisure activity and wellbeing in later life. Ageing Soc 2011;31:683-712.

5 Kolanowski A, Buettner L, Costa P. Capturing interests: therapeutic recreation activities for persons with dementia. Ther Recreation $J$ 2001;35:220-36 https://js.sagamorepub.com/trj/article/view/1062

6 Kasper B, Lubecki U. Zu Fuss unterwegs - mobilität und freizeit älterer menschen. Raum und Mobilität Arbeitspapiere des Fachgebiets Verkehrswesen und Verkehrsplanung 2003;10:1-12 http://www.vpl.tu-dortmund.de/cms/Medienpool/PDF_Dokomunte/ Arbeitspapiere/AP10_von_Birgit_Kasper_und_Ulrike_Lubecki.pdf

7 Thomas JE, O'Connell B, Gaskin CJ. Residents' perceptions and experiences of social interaction and participation in leisure activities in residential aged care. Contemp Nurse 2013;45:244-54.

8 Buettner LL, Fitzsimmons S. Activity calendars for older adults with dementia: what you see is not what you get. Am J Alzheimers Dis Other Demen 2003;18:215-26.

9 Wong C, Leland N. Perspectives of nursing home residents and staff on barriers and facilitators to activity engagement. Innov Aging 2018;2:538-9.

$10 \mathrm{Li} \mathrm{L}$, Chang H-J, Yeh H-I, et al. Factors associated with leisure participation among the elderly living in long-term care facilities. Int $J$ Gerontol 2010;4:69-74.

11 Jenkins J, Felce D, Lunt B, et al. Increasing engagement in activity of residents in old people's homes by providing recreational materials. Behav Res Ther 1977;15:429-34.

12 Tak SH, Kedia S, Tongumpun TM, et al. Activity engagement: perspectives from nursing home residents with dementia. Educ Gerontol 2015;41:182-92.

13 Büker C. Das Potenzial der tagespflege - eine chance für pflegebedürftige, angehörige und die professionelle pflege. Pflegezeitschrift 2017;70:20-3 http://www.christa-bueker.de/ Publikationen

14 Büker C, Niggemeier M. Tagespflege für ältere menschen: ein praxisbuch. Stuttgart: W. Kohlhammer GmbH, 2014.

15 Teufel J. Standards für einrichtungen der tagespflege: betreuung und beschäftigung. Hannover: Schlütersche, 2011.

16 Gaugler JE, Marx K, Dabelko-Schoeny H, et al. COVID-19 and the need for adult day services. J Am Med Dir Assoc 2021;22:1333-7.

17 Jopp DS, Hertzog C. Assessing adult leisure activities: an extension of a self-report activity questionnaire. Psychol Assess 2010;22:108-20.

18 Stacke TI, Bergmann JM, Ströbel AM, et al. Preferences for everyday living inventory (peli): study protocol for piloting a culture-sensitive and setting-specific translated instrument in German care settings (PELI-D). BMJ Open 2020;10:e030268.

19 Van Haitsma K, Curyto K, Spector A, et al. The preferences for everyday living inventory: scale development and description of psychosocial preferences responses in community-dwelling elders. Gerontologist 2013;53:582-95.

20 Cohen-Mansfield J, Jensen B. Self-maintenance habits and preferences in elderly (shape): reliability of reports of self-care preferences in older persons. Aging Clin Exp Res 2007;19:61-8.

21 Rommerskirch-Manietta M, Roes M, Palm R. Präferenzen des alltäglichen Lebens in Der Pflegedokumentation - eine Dokumentenanalyse in verschiedenen pflegerischen settings. Pflege 2021;34:191-202.

22 Rommerskirch-Manietta M, Roes M, Stacke T-I, et al. Präferenzen von menschen MIT Pflegebedarf. HeilberufeScience 2021;12:13-21.

23 Stacke TI, Manietta C, Purwins D. Übersetzung und kultursensitive anpassung des preferences for everyday living Inventory(C) für pflegerische Versorgungssettings. Pflege 2021;3.

24 Roes M, Stacke T, Bergmann J, et al. Difficulties within the culturally sensitive translation of the peli. Innov Aging 2018;2:43.

25 Miake-Lye IM, Hempel S, Shanman R, et al. What is an evidence MAP? a systematic review of published evidence maps and their definitions, methods, and products. Syst Rev 2016;5:28.

26 Kane M, Trochim WM. Concept mapping for planning and evaluation - applied social research methods. Thousand Oaks, California: Sage Publications, Inc, 2007.

27 DeVon HA, Block ME, Moyle-Wright P, et al. A psychometric toolbox for testing validity and reliability. J Nurs Scholarsh 2007;39:155-64.

28 O'Brien BC, Harris IB, Beckman TJ, et al. Standards for reporting qualitative research: a synthesis of recommendations. Acad Med 2014;89:1245-51.

29 Wright MT. Partizipative qualitätsentwicklung in der gesundheitsförderung und prävention. Bern: Huber, 2010.

30 Bergold J, Thomas S. Partizipative Forschung. In: Mey G, Mruck $\mathrm{K}$, eds. Handbuch qualitative Forschung in Der Psychologie. Wiesbaden: VS Verlag für Sozialwissenschaften, 2010: 333-44. 
31 Arksey H, O'Malley L. Scoping studies: towards a methodological framework. Int J Soc Res Methodol 2005;8:19-32.

32 Levac D, Colquhoun H, O'Brien KK. Scoping studies: advancing the methodology. Implementation Science 2010;5:1-9.

33 Rommerskirch-Manietta M, Purwins D, Van Haitsma K, et al. Instruments for assessing the preferences for everyday living of older people with various care needs: protocol for an evidence MAP. BMJ Open 2021;11:e048921-5.

34 Van Haitsma K, Abbott KM, Heid AR, et al. The consistency of selfreported preferences for everyday living: implications for personcentered care delivery. J Gerontol Nurs 2014;40:34-46.

35 Stacke TI, Manietta C, Bergmann JM. Übersetzung und kultursensitive anpassung des peli (preferences for everyday living inventory) für den Einsatz in verschiedenen pflegerischen settings. Pflege 2021.

36 Koo TK, Li MY, . A guideline of selecting and reporting intraclass correlation coefficients for reliability research. $J$ Chiropr Med 2016;15:155-63.

37 Cicchetti DV. Guidelines, criteria, and rules of thumb for evaluating normed and standardized assessment instruments in psychology. Psychol Assess 1994;6:284-90.

38 Van Haitsma K, Kleban M, Curyto K. Final report assessing preferences for everyday living in the nursing home. Reliability and Concordance Issues 2014;199 https://scholar.google.com/scholar lookup?author=KS+Van+Haitsma\&author=M+Kleban\&author= $\mathrm{KJ}+$ Curyto\&title=Assessing+preferences+for+everyday+living+ in+the+nursing+home $\% 3 \mathrm{~A}+$ reliability+and+concordance+issues\& publication_year=2014

39 Bujang MA, Baharum N. A simplified guide to determination of sample size requirements for estimating the value of intraclass correlation coefficient: a review. Archives of Orofacial Scien 2017;12:1-11 https://www.researchgate.net/publication/318788161 A_simplified_guide_to_determination_of_sample_size_requirements for estimating the value of intraclass correlation coefficient $A$ review

40 Walter SD, Eliasziw M, Donner A. Sample size and optimal designs for reliability studies. Stat Med 1998:17:101-10.

41 Schatz T, Haberstroh J, Bindel K, et al. Improving comprehension in written medical informed consent procedures. GeroPsych 2017;30:97-108
42 Hopf C. Flick U, von Kardorff E, Steinke I, eds. Qualitative forschung: ein Handbuch. Hamburg: Rowohlt Taschenbuch Verlag, 2015: 349-60.

43 Helfferich C. Die qualität qualitativer daten - manual für die durchführung qualitativer interviews. Wiesbaden: VS Verlag für Sozialwissenschaften, 2009.

44 Dresing T, Pehl T, Transkription P. Regelsysteme, software und praktische anleitungen für qualitative forscherinnen. Marburg: Eigenverlag, 2018.

45 Trochim WM, McLinden D. Introduction to a special issue on concept mapping. Eval Program Plann 2017;60:166-75.

46 Systems C. Concept systems Incorparated. secondary concept systems Incorparated, 2020. Available: https://www.conceptsystems. com/home

47 MAXQDA. Software für qualitative datenanalyse. Berlin, Deutschland: Consult Sozialforschung GmbH, 2021: 1989.

48 Elo S, Kääriäinen M, Kanste O, et al. Qualitative content analysis. Sage Open 2014;4:215824401452263.

49 Elo S, Kyngäs $\mathrm{H}$. The qualitative content analysis process. J Adv Nurs 2008;62:107-15.

50 GBIF. A language and environment for statistical computing. Vienna, Austria, 2020.

51 Matheson GJ. We need to talk about reliability: making better use of test-retest studies for study design and interpretation. PeerJ 2019;7:e6918.

52 Landis JR, Koch GG. The measurement of observer agreement for categorical data. Biometrics 1977;33:159-74.

53 WMA. WMA Declaration of Helsinki - ethical prinicples for medical research involving human subjects. secondary wma declaration of helsinki - ethical prinicples for medical research involving human subjects, 2018. Available: https://www.wma.net/policies-post/ wma-declaration-of-helsinki-ethical-principles-for-medical-researchinvolving-human-subjects/

54 Schnell MW, Dunger C. Forschungsethik. Informieren-reflektierenanwenden. In: vollständige überarbeitete und erweiterte Auflage. Vol 2. Bern: Hogrefe Verlag, 2018.

55 Dewing J. Participatory research - A method for process concent with persons who have dementia. Dementia 2016;6:11-25. 